\title{
Electrical performance of InAs/AlSb/GaSb superlattice photodetectors
}

\author{
T. Tansel ${ }^{a,}{ }^{*}$, M. Hostut ${ }^{b}$, S. Elagoz ${ }^{\text {c }}$, A. Kilic ${ }^{d}$, Y. Ergun ${ }^{d}$, A. Aydinli ${ }^{e}$ \\ a Institute of Nuclear Sciences, Hacettepe University, Turkey \\ ${ }^{\mathrm{b}}$ Div. of Physics Education, Faculty of Education, Akdeniz University, Turkey \\ ${ }^{\mathrm{c}}$ Department of Nanotechnology Eng., Cumhuriyet University, Turkey \\ d Department of Physics, Anadolu University, Turkey \\ e Department of Physics, Bilkent University, Turkey
}

\section{A R T I C L E I N F O}

\section{Article history:}

Received 17 November 2015

Received in revised form 22 December 2015

Accepted 23 December 2015

Available online 29 December 2015

\section{Keywords:}

Superlattice

InAs/AlSb/GaSb based T2SL N-structures

with AlSb

Mid wavelength infrared

\begin{abstract}
A B S T R A C T
Temperature dependence of dark current measurements is an efficient way to verify the quality of an infrared detector. Low dark current density values are needed for high performance detector applications. Identification of dominant current mechanisms in each operating temperature can be used to extract minority carrier lifetimes which are highly important for understanding carrier transport and improving the detector performance. InAs/AlSb/GaSb based T2SL N-structures with AlSb unipolar barriers are designed for low dark current with high resistance and detectivity. Here we present electrical and optical performance of such $\mathrm{N}$-structure photodetectors.
\end{abstract}

() 2016 Elsevier Ltd. All rights reserved.

\section{Introduction}

InAs/AlSb/GaSb based superlattice (SL) material system known as 6.1A material family is highly desirable for designing new high performance photodetectors operating in the mid wavelength infrared range (MWIR). Depending on the doping concentrations and configuration of the constituent alloys of InAs, AlSb and GaSb in the superlattice period, SL band structure may be adjusted in order to improve electrical and optical performance of photodetectors. There have been a number of high performance type-II SL detector architectures reported up to date. These include nBn, pBp, XBn, CBIRD, pBIBn, W and M structures and Ga free InAs/InAsSb based type-II SL [1,2]. The details of the barrier detector structures are reviewed by Martyniuk et al. [3]. Recently, we proposed new detector architecture called N structure which is a pin photodiode with AlSb unipolar electron barriers. N structure aims to improve the overlap of spatially separated electron and hole wave functions. The layer configurations and energy band alignment of the structure are shown in Fig. 1 (a) and (b), respectively. In the detector structure, thin AlSb layers are placed in between InAs and GaSb layers. Under reverse bias AlSb barriers push the carriers towards $\mathrm{GaSb} / \mathrm{InAs}$ interfaces to increase electron and hole wave function overlap enhancing type-II optical transition (Fig. 2). Compare to standard InAs/GaSb SL detectors, the overlap of carrier wave functions is increased by about $25 \%$ with $\mathrm{N}-$ structure design [4]. The specific detectivity in these detectors was measured as high as $3 \times 10^{12}$ Jones with cut-off wavelengths of $4.3 \mu \mathrm{m}$ at $79 \mathrm{~K}$ reaching to $2 \times 10^{9}$ Jones and $4.5 \mu \mathrm{m}$ at $255 \mathrm{~K}$ [5].

\footnotetext{
* Corresponding author.

E-mail addresses: tunaytansel@hacettepe.edu.tr (T. Tansel), mhostut@akdeniz.edu.tr (M. Hostut), elagoz@cumhuriyet.edu.tr (S. Elagoz), abkilic@anadolu. edu.tr (A. Kilic), yergun@anadolu.edu.tr (Y. Ergun), aydinli@fen.bilkent.edu.tr (A. Aydinli).
} 


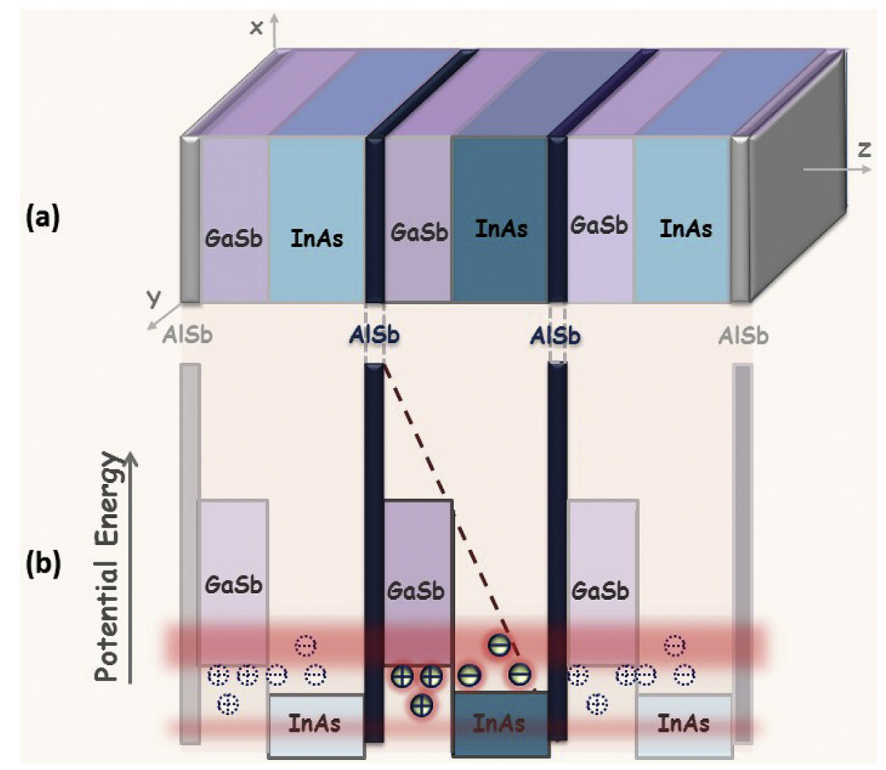

Fig. 1. (a) Layer sequence in growth direction, (b) conduction and valence band profiles for asymmetric InAs/AsSb/GaSb based T2SL N-structure. (Electron and hole minibands are given by shady red). (For interpretation of the references to colour in this figure legend, the reader is referred to the web version of this article.)

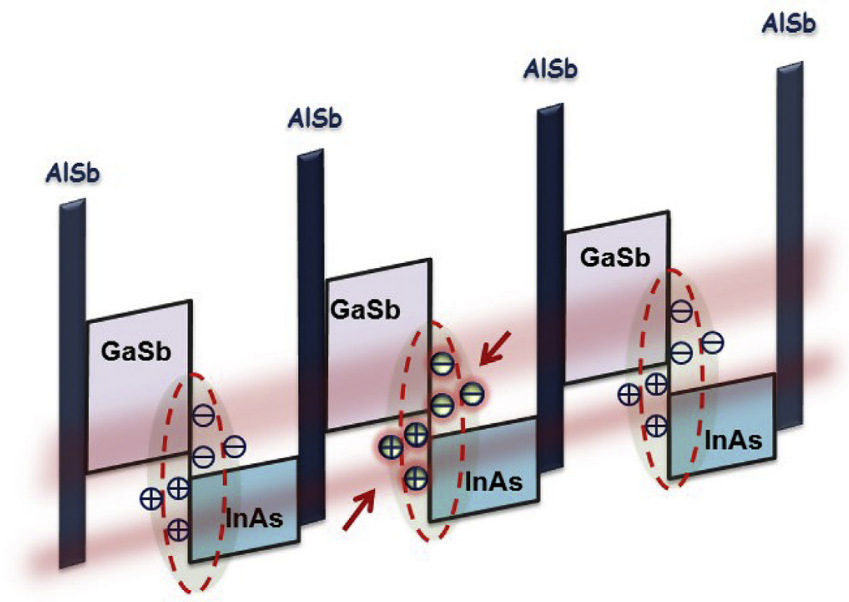

Fig. 2. Conduction and valence band profiles for N-structure with electron and hole confinement under reverse bias.

On the other hand, lower dark current densities are needed in order to achieve high performance focal plane arrays (FPAs). The identification of dark current mechanisms in an SL structure such as diffusion, generation-recombination (GR), band to band tunneling (BTB), trap assisted tunneling (TAT) currents and extracted carrier lifetimes are very important parameters for understanding of the transport mechanism and improving the detector performance. In the literature, temperature dependent performance in a short period InAs/GaSb pin SL photodiode within the MWIR domain has been investigated [6] to identify dominant dark current components. Minority carrier lifetimes were observed in the range between 5 and 100 ns. Better results on carrier lifetimes of $>412$ ns have been obtained on Ga free InAs/InAsSb SL structures by time resolved photoluminescence measurements [7]. Vertical transport parameters such as vertical carrier mobilities and carrier concentrations are directly related to drift and diffusion of excited carriers through the SL growth layers and are useful for identification of high temperature detector performance. Szmulowicz et al. advanced a theoretical model for calculation of vertical and in-plane carrier mobilities in InAs/GaSb SL [8]. Umana-Membreno et al. reported the results of an experimental study of the vertical carrier mobility in InAs/GaSb T2SL using variable magnetic field geometric magneto-resistance measurements [9]. 
In this study, we report current density-voltage $(\mathrm{J}-\mathrm{V})$ characteristics of InAs/AlSb/GaSb based type-II SL N-structure photodiodes as a function of temperature (87-271 K). We, then, fit dark current densities by using Shockley Formula to extract minority carrier lifetimes at different temperatures.

\section{Dark current model}

The analysis of the dark current contributions is made by using well known Shockley Model [10]. The main influence on dark current components is taken into account as three terms: i) diffusion of thermally generated minority carriers from the quasi-neutral regions, ii) generation-recombination of carriers in the depletion region and iii) trap assisted tunnelling carriers. The band-to-band tunneling current $J_{B T B}$ has negligible influence on both forward and reverse biased characteristics while trap assisted tunnelling current $J_{T A T}$ only appears at reverse bias. Therefore the total current for.

InAs/AlSb/GaSb based type-II SL detectors is given by:

$$
J_{T}=J_{D I F F}+J_{G R}+J_{T A T}
$$

Diffusion current occurs from thermal excitation of minority carriers, located inside the quasi-neutral regions, diffusing across the junction in order to maintain charge neutrality. In order for this mechanism to be effective, carriers must be within a diffusion length [11]. Diffusion current is given as:

$$
J_{\text {DIFF }}=q\left[\frac{L_{e} n_{i}^{2}}{\tau_{e} N_{A}}+\frac{L_{h} n_{i}^{2}}{\tau_{h} N_{D}}\right]\left(\operatorname{Exp}\left[\frac{q V}{k T}\right]-1\right)
$$

where $q$ is electronic charge, $V$ is bias voltage, $L_{e}=\sqrt{D_{e} \tau_{e}}$ and $L_{h}=\sqrt{D_{h} \tau_{h}} ; D_{e}=\frac{k T}{q} \mu_{e}$ and $D_{h}=\frac{k T}{q} \mu_{h} ; L_{e}, L_{h}$ and $D_{e}, D_{h}$ are electron, hole diffusion lengths and diffusion constants, $\tau_{e}, \tau_{h}$ and $\mu_{e}, \mu_{h}$ are electron, hole diffusion lifetimes and mobilities respectively, $n_{i}$ is the intrinsic carrier concentration in the InAs/AlSb/GaSb SL given as $n_{i}=2\left[2 \pi k / h^{2}\right]^{3 / 2}\left(m_{e} m_{h}\right)^{3 / 4} \operatorname{Exp}\left(-E_{g} / 2 k T\right), k$ is Boltzman's constant, $T$ is the temperature, $E_{g}$ is the SL band gap energy and $m_{e}, m_{h}$ are electron and hole effective masses. $N_{D}$ and $N_{A}$ are donor and acceptor concentrations in $\mathrm{n}$ and $\mathrm{p}$ side of the pn junction. We assume that the nid SL zone of the junction is residually p-type and electrons are the main minority carriers leading to diffusion current with $\tau_{e}$ lifetime. The contribution of hole diffusion current has been ignored in our calculations. Therefore, Eqn. (2) becomes,

$$
J_{D I F F}=q\left[\frac{L_{e} n_{i}^{2}}{\tau_{e} N_{A}}\right]\left(\operatorname{Exp}\left[\frac{q V}{k T}\right]-1\right)
$$

The nid thickness $(\mathrm{W}=0.36 \mu \mathrm{m})$ is smaller than electron diffusion length. In the calculations $N_{D}$ is equal to $5 \times 10^{17} \mathrm{~cm}^{-3}$. Temperature dependence of $N_{A}$ in the absorber is in the range of $1 \times 10^{15}-1 \times 10^{16} \mathrm{~cm}^{-3}$ [8,12] and mobilities are taken as vertical mobilities [13].

Generation-recombination (GR) current originates from the GR centres (also known as Shockley-Read-Hall traps) in the middle of the band gap of depletion region causing the generation-recombination of minority carriers. A reverse bias applied to the diode activates these G-R centres. As a result, electron-hole pairs are generated out of the valence band or into the conduction immediately removed by the electric field of the depletion region by giving rise to the GR current. GR current is given by the following expressions:

$$
\begin{aligned}
& J_{G R}=\frac{q n_{i} W}{\tau_{G R}} \frac{2 k T}{q\left(V_{b i}-V\right)} \operatorname{Sinh}\left(\frac{q V}{2 k T}\right) f(b) \\
& f(b)=\left\{\begin{array}{lr}
\frac{1}{\sqrt[2]{b^{2}-1}} \ln \left(2 b^{2}+2 b \sqrt{b^{2}-1}-1\right) & b<1 \\
1 & b=1 \\
\frac{1}{\sqrt{1-b^{2}}} \operatorname{Tan}^{-1}\left(\frac{\sqrt{1-b^{2}}}{b}\right) & b>1
\end{array}\right. \\
& b=\operatorname{Exp}(-q V / 2 k T) \operatorname{Cosh}\left(\frac{E_{t}-E_{i}}{k T}\right)
\end{aligned}
$$

where $\tau_{G R}$ is the GR lifetime, $V_{b i}$ is the built-in voltage and $E_{t}$ and $E_{i}$ are trap and intrinsic energy levels in the band gap.

The last current component is trap assisted tunnelling current which originates from minority carriers occupying the trap states in or near the depletion region and tunnelling across the junction. This mechanism includes a thermally activated transition of carriers from the valence band to the trap site, and a zero energy tunnel into an empty state in the conduction 
Table 1

Material parameters used in the calculation of dark current densities at various temperatures.

\begin{tabular}{lllr}
\hline Temp. (K) & $\mathrm{N}_{\mathrm{A}}\left(\mathrm{cm}^{-3}\right)$ & $\mathrm{Eg}(\mathrm{eV})$ & $\tau_{e}(n s)$ \\
\hline 87 & $5.8 \times 10^{15}$ & 0.283 & 1 \\
100 & $6.0 \times 10^{15}$ & 0.281 & 1 \\
140 & $6.5 \times 10^{15}$ & 0.275 & 40 \\
160 & $7 \times 10^{15}$ & 0.272 & 35 \\
189 & $8 \times 10^{15}$ & 0.267 & 70 \\
215 & $9 \times 10^{15}$ & 0.262 & 110 \\
\hline
\end{tabular}

band. TAT current component, which is about four orders of magnitude lower than GR current, has negligible influence on our calculations. The TAT current can be stated as [11,14]:

$$
J_{T A T}=\frac{q^{2} m_{e}^{*} V M^{2} N_{t}}{8 \pi \hbar^{3}} \operatorname{Exp}\left[\frac{-4 \sqrt{2 m_{e}^{*}\left(E_{g}-E_{t}\right)^{3}}}{3 q \hbar E}\right]
$$

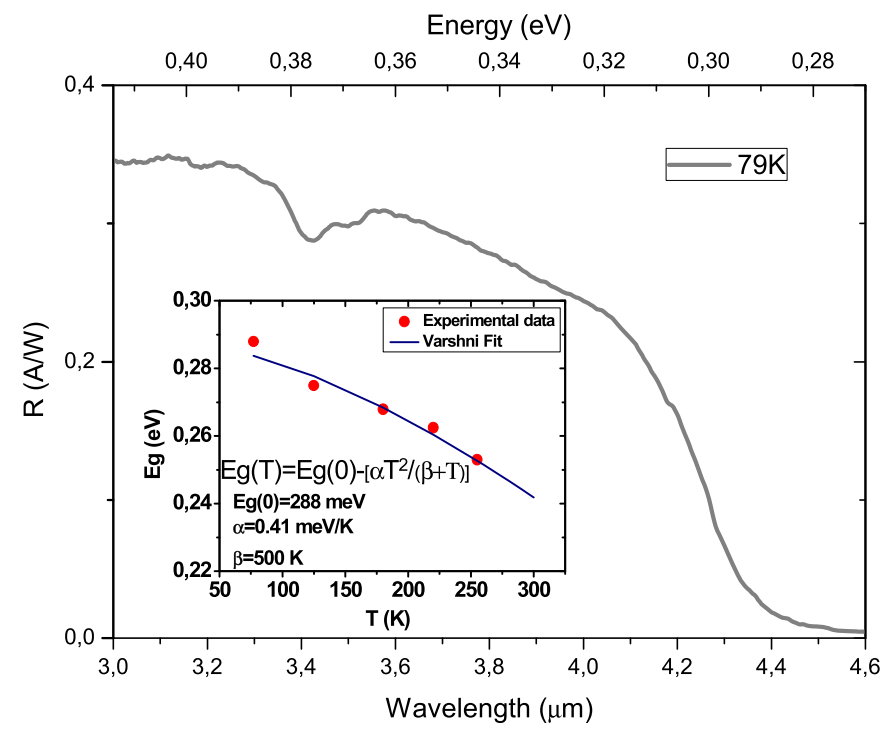

Fig. 3. Responsivity spectrum of $\mathrm{N}$-Structure at $79 \mathrm{~K}$. Inset shows Varshni fit for band gap energy extracted from optical response spectra for different temperatures.
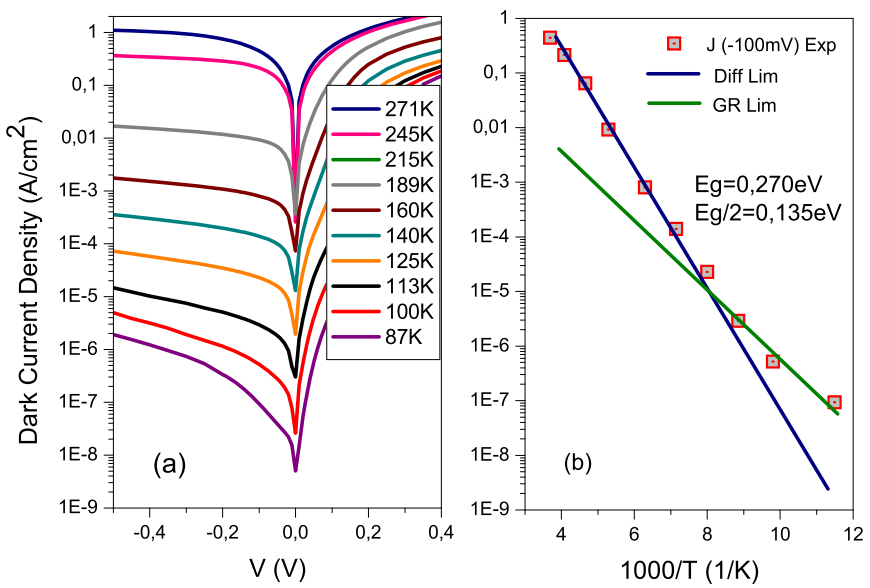

Fig. 4. (a) Temperature dependence of dark current density, (b) Arrhenius plot of dark current density vs inverse temperature (1000/T). 

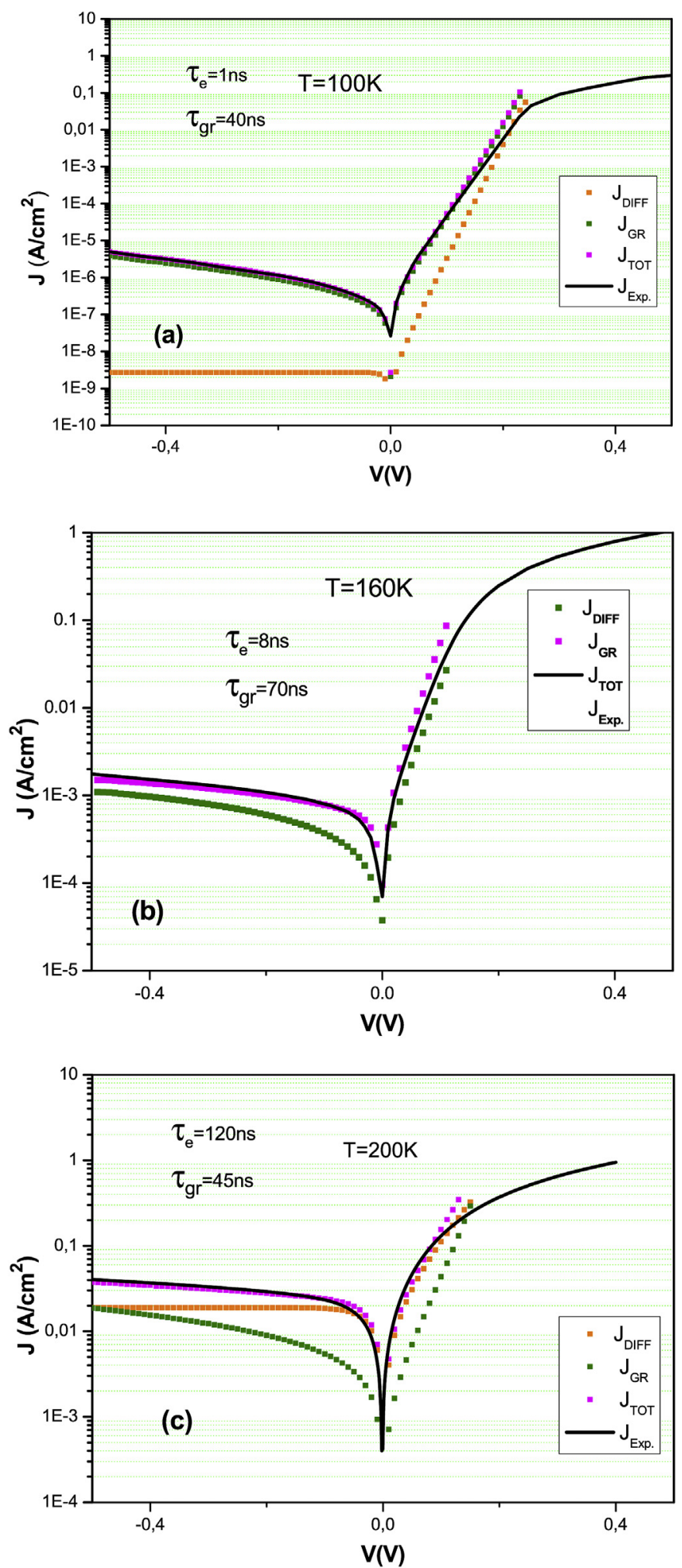

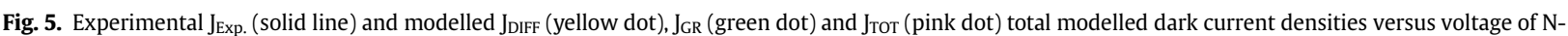
structure SL photodiode at (a) $\mathrm{T}=100 \mathrm{~K}$, (b) $\mathrm{T}=160 \mathrm{~K}$ and (c) $\mathrm{T}=200 \mathrm{~K}$. (For interpretation of the references to colour in this figure legend, the reader is referred to the web version of this article.) 
where $m_{e}^{*}$ is the tunnelling electron effective mass, $M$ is the matrix element associated with the trap potential assumed to be $10^{-23} \mathrm{eV}^{2} \mathrm{~cm}^{3}, N_{t}$ is the trap density equal to $2 \times 10^{11} \mathrm{~cm}^{-3}, \hbar$ is Planck's constant, $E$ is the electric field strength across the depletion region. Table 1 shows some detector parameters for calculations of dark current density.

\section{Experimental results}

The superlattice photodiode was grown by commercially (IQE Inc. USA) with molecular beam epitaxy. First a $100 \mathrm{~nm}$ GaSb buffer layer is deposited on unintentionally p-type doped (100) GaSb substrate followed by a $20 \mathrm{~nm}$ lattice matched $\mathrm{Al}_{0,4} \mathrm{Ga}_{0,6} \mathrm{As}_{0,04} \mathrm{Sb}_{0,96}$ buffer layer. $1000 \mathrm{~nm}$ thick p-type GaSb: Be $\left(\mathrm{p}=1 \times 10^{17} \mathrm{~cm}^{-3}\right.$ ) bottom contact is grown on the buffer layer. The p-i-n detector structure consists of 9/2/8.5 MLs) of InAs/AlSb/GaSb SL layers as 90 periods of p-region with GaSb: Be $\left(\mathrm{p}=1.5 \times 10^{17} \mathrm{~cm}^{-3}\right), 60$ period of $\mathrm{i}$-intrinsic region and 40 periods of n-region with $\mathrm{InAs}$ : Te $\left(\mathrm{n}=5 \times 10^{17} \mathrm{~cm}^{-3}\right)$. The device is terminated by $20 \mathrm{~nm}$ InAs: Te $\mathrm{n}$-contact $\left(\mathrm{n}=5 \times 10^{17} \mathrm{~cm}^{-3}\right)$. Standard lithography was used to define square mesas with different dimensions. The fabrication details are given elsewhere [15]. The J-V curves are fitted by using Shockley Formula in order to identify the dominant dark current mechanism in each operating temperature range. We then extracted minority carrier lifetimes of the MWIR SL photodiode quantitatively [16]. We measured responsivity spectra of the detector at various temperatures. Fig. 3 shows the responsivity spectrum of the structure at $79 \mathrm{~K}$. The device gives $50 \%$ cut-off wavelengths at $4.2 \mu \mathrm{m}$.

In this design, we use the detector structure with short period of absorption layer in order to measure minority carrier lifetimes. For this structure, the peak responsivity of $0.35 \mathrm{~A} / \mathrm{W}$ at $3 \mu \mathrm{m}$ in wavelength can be regarded as low at a short period detector with absorption layer thickness of $0.36 \mu \mathrm{m}$ but, if the absorption layer thickness is increased four times, the quantum efficiency and photoresponse will be expected to increase theoretically [17].

Temperature dependence of the band gap energy $\mathrm{Eg}(\mathrm{T})$ of the SL structure is extracted from responsivity spectra [5], where data are taken at 50\% cut-off wavelengths, measured in the range of 79-255 K by fitting Varshni's equation shown in inset of Fig. 3.

Dark current density-voltage measurements are carried out under dark conditions for different operating temperatures ranging from $87 \mathrm{~K}$ to $271 \mathrm{~K}$ using HP41420A source measure unit. Fig. 4 (a) shows the dark current density-voltage characteristics of $500 \times 500 \mu \mathrm{m}^{2}$ diodes under applied bias voltage range [-0.5 V, $\left.0.5 \mathrm{~V}\right]$. At $87 \mathrm{~K}$ and under $-0.1 \mathrm{~V}$ bias voltage, the dark current density is measured as $9.29 \times 10^{-8} \mathrm{~A} / \mathrm{cm}^{2}$ and corresponding to a dynamic resistance area product (RA) determined as $6.43 \times 10^{5} \Omega \mathrm{cm}^{2}$. These values are very promising for low temperature applications. Extracted from Fig. 4 (a), the inverse temperature $(1000 / \mathrm{T})$ dependence of dark current density under $-100 \mathrm{mV}$ bias is shown in Fig. 4 (b). In the temperature range $271-125 \mathrm{~K}$, the dark current density reveals diffusion-limited behaviour (Arrhenius type) with associated activation energy of $270 \mathrm{meV}$ which is close to the band gap energy. In the lower temperature range (100-80 K), the dominant mechanism starts to become generation recombination (GR) which mostly depends on the deep trap levels inside the band gap (Eg/2). To illustrate the bias dependent dominant dark current components of diffusion (JDIFF) and GR (JGR) current, we use the model given elsewhere [14]. We then fit the dark current densities to determine the minority carriers of diffusion and GR lifetimes. The experimental dark current density data and modelled data for dark current density components at $\mathrm{T}=100 \mathrm{~K}, 160 \mathrm{~K}$ and $200 \mathrm{~K}$ are shown in Fig. 5(a) to (c) with fitting parameters of minority carrier lifetimes. At $100 \mathrm{~K}$ (Fig. 5(a)), GR current dominates the dark current due to low p-type background carrier density of depletion region, while diffusion current dominates the dark current at $200 \mathrm{~K}$ with an increased diffusion carrier lifetime of 120 ns due to an

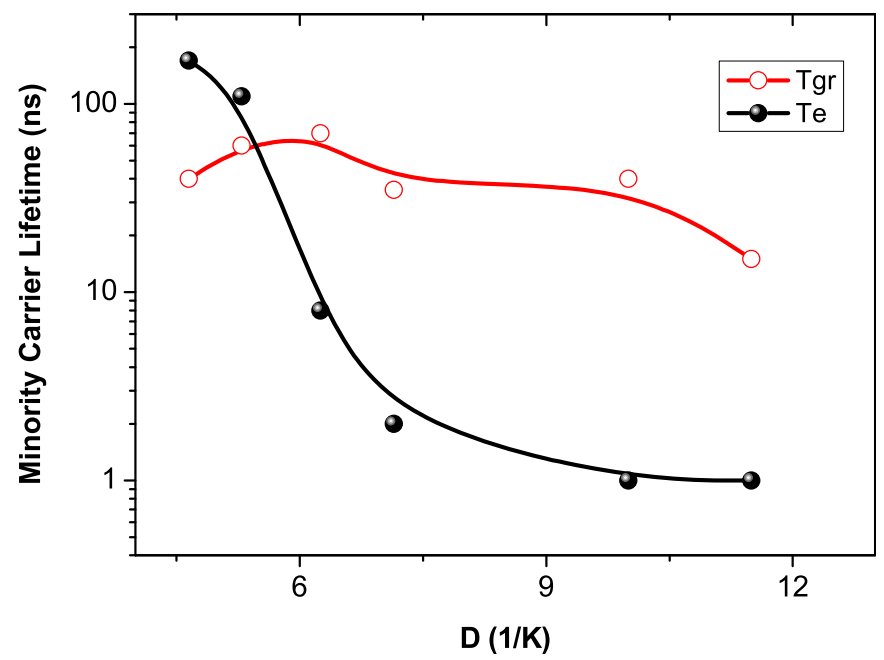

Fig. 6. Calculated diffusion $\left(\tau_{\mathrm{e}}\right)$ and $\mathrm{GR}\left(\tau_{\mathrm{GR}}\right)$ lifetimes at various temperatures 
increased carrier density with temperature (Fig. 5(c)). At $160 \mathrm{~K}$, dark current is influenced both diffusion and GR components of dark current with determined of the minority carrier lifetimes $\tau_{e}=8 \mathrm{~ns}$ and $\tau_{G R}=70 \mathrm{~ns}$ given in Fig. 5 (b). We have also calculated minority lifetimes at various temperatures given by Fig. 6 . While diffusion lifetimes are increased with increasing temperature ranging from 1 to $120 \mathrm{~ns}$, GR lifetimes mostly behave independent of temperature.

\section{Conclusions}

Temperature dependence of $\mathrm{J}-\mathrm{V}$ characteristics of InAs/AlSb/GaSb based T2SL N-structures are analysed. Deduced from $\mathrm{J}-\mathrm{V}$ curve-fitting, minority carrier lifetimes have been estimated in the temperature range $87-215 \mathrm{~K}$. At $87 \mathrm{~K}$ and under $-0.1 \mathrm{~V}$ bias voltage, the dark current density is measured as $9.29 \times 10^{-8} \mathrm{~A} / \mathrm{cm}^{2}$ and corresponding dynamic resistance area product (RA) is determined as $6.43 \times 10^{5} \Omega \mathrm{cm}^{2}$. In the temperature range $271-125 \mathrm{~K}$, the dark current density reveals diffusion-limited behaviour (Arrhenius type) with electron lifetime values between $1 \mathrm{~ns}$ and $120 \mathrm{~ns}$. In the lower temperature range (125-87 K), the dominant mechanism starts to become generation recombination (GR) with GR lifetimes varying between 15 and $70 \mathrm{~ns}$.

\section{Acknowledgements}

Y.Ergun and A. Kilic acknowledge the supports of Anadolu University (BAP Grant: 13005F108). Author M. Hostut also acknowledges the supports of Akdeniz University (BAP Grant: FKA-2015-918).

\section{References}

[1] T. Schuler-Sandy, S. Myers, B. Klein, N. Gautam, P. Ahirwar, Z.-B. Tian, T. Rotter, G. Balakrishnan, E. Plis, S. Krishna, Appl. Phys. Lett. 101 (2012) 071111.

[2] H.S. Kim, O.O. Cellek, Zhi-Yuan Lin, Zhao-Yu He, Xin-Hao Zhao, Shi Liu, H. Li, Y.-H. Zhang, Appl. Phys. Lett. 101 (2012) 161114.

[3] P. Martyniuk, M. Kopytko, A. Rogalski, Opto-Electron. Rev. 22 (2014) 127-146.

[4] O. Salihoglu, A. Muti, K. Kutluer, T. Tansel, R. Turan, Y. Ergun, A. Aydinli, Appl. Phys. Lett. 101 (2012) 073505.

[5] M. Hostut, M. Alyoruk, T. Tansel, A. Kilic, R. Turan, A. Aydinli, Y. Ergun, N-structure based on InAs/AlSb/GaSb superlattice photodetectors, Superlattices Microstruct. 79 (2015) 116-122.

[6] C. Cervera, K. Jaworowicz, H. Aït-Kaci, R. Chaghi, J.B. Rodriguez, I. Ribet-Mohamed, P. Christol, Infrared Phys. Technol. 54 (2011) $258-262$.

[7] E.H. Steenbergen, B.C. Connelly, G.D. Metcalfe, H. Shen, M. Wraback, D. Lubyshev, Y. Qiu, J.M. Fastenau, A.W.K. Liu, S. Elhamri, O.O. Cellek, Y.-H. Zhang, Appl. Phys. Lett. 99 (2011) 251110.

[8] F. Szmulowicz, G.J. Brown, Appl. Phys. Lett. 98 (2011) 182105.

[9] G.A. Umana-Membreno, B. Klein, H. Kala, J. Antoszewski, N. Gautam, M.N. Kutty, E. Plis, S. Krishna, L. Faraone, Appl. Phys. Lett. 101 (2012) 253515.

[10] C.-T. Sah, R.N. Noyce, W. Shockley, Proc. IRE. 45 (1957) 1228-1243.

[11] J. Nguyen, D.Z. Ting, C.J. Hill, A. Soibel, S.A. Keo, S.D. Gunapala, Infrared Phys. Technol. 52 (2009) 317-321.

[12] C. Asplund, R.M. von Würtemberg, D. Lantz, H. Malm, H. Martijn, E. Plis, N. Gautam, S. Krishna, Infrared Phys. Technol. 59 (2013) $22-27$.

[13] H.J. Haugan, S. Elhamri, G.J. Brown, W.C. Mitchel, J. Of Appl. Phys. 104 (2008) 073111.

[14] C. Cervera, J.B. Rodriguez, R. Chaghi, H. Ait-Kaci, J.B. Rodriguez, P. Christol, J. Appl. Phys. 106 (2009) 024501.

[15] O. Salihoglu, A. Muti, K. Kutluer, T. Tansel, R. Turan, A. Aydinli, J. Appl. Phys. 111 (2012) 074509.

[16] C. Cerveva, I. Ribet-Mohamed, R. Taalat, J.P. Perez, P. Christol, J.B. Rodriguez, J Electron. Mater. 41 (2012) 2714-2718.

[17] R. Rehm, M. Walther, J. Schmitz, F. Rutz, J. Fleißner, Ralf Scheibner, J. Ziegler, Infrared Phys. Technol. 52 (2009) 344-347. 